\title{
EL LADO OSCURO DE LA MUJER EN LA INVESTIGACIÓN CIENTÍFICA: ¿ES LA CIENCIA UNA "EMPRESA" MASCULINA?
}

Yolanda Agudo Arroyo

Universidad Nacional de Educación a Distancia

\section{Introducción}

Con el empeño de reflexionar lejos de las conjeturas comúnmente establecidas en la sociedad y de analizar tomando distancia de las mismas, antes de evidenciar los velos y oscuridades que adquiere lo femenino en el ámbito científico, es necesario vislumbrar qué se entiende por ciencia para poder comprender los debates que han surgido alrededor de este término. A este respecto, con el riesgo de simplificar, podemos aseverar que la función social que desempeña la ciencia es la de obtener conocimiento a través de la exploración del mundo y la investigación de la naturaleza. Esta función se desempeña con los métodos que deciden usar los miembros de la comunidad científica para alcanzar dicho conocimiento, de manera que sus resultados puedan ser evaluados e incluso criticados (Pérez Sedeño, 2000b).

Allá por el siglo XVI, con la filosofía baconiana, se apuntaba que la actividad científica debía ser impersonal y objetiva; asimismo debía utilizar un método que garantizara la verdad y además, de acuerdo con esta filosofía, debía asentarse en el dominio de la naturaleza. Pues bien, es claro que la actividad científica, supuestamente basada en un conocimiento técnico-impersonal, adquirió una función central con la revolución industrial del siglo XIX, llegando a condicionar la cultura y el modo de pensar. 
Cuanto menos, con la rapidez del adelanto tecnológico, proporcionado por la revolución industrial, se hizo evidente la importancia del conocimiento científico. El criterio científico se ha ido modificado desde sus comienzos hasta nuestros días a través de un proceso de transformación del pensamiento y de la visión del mundo. Tal proceso suscita que la "ciencia moderna" se presente como impersonal y mecánica, basada en unos principios dominantes del método científico cada vez más distanciados de la teleología.

Ahora bien, a este respecto, y siguiendo a Durán, nos percatamos de la ineludible crítica a los supuestos teóricos que se le atribuyen a la ciencia. Ésta reconoce que no cabe la menor duda de que las atractivas cualidades que ahora se atribuyen a la ciencia moderna, en otras épocas se asignaron a lo divino. Ante la población no iniciada, la ciencia y los científicos, dice, se han cubierto de un ropaje impenetrable que les separa y protege, con todo, advierte, hemos de ser conscientes de que la ciencia la han construido grupos específicos para fines igualmente específicos (Durán, 1996).

Desde su reaparición ${ }^{1}$ como institución objetiva, analítica y neutral, a lo largo del tiempo la ciencia se ha ido complejizando, especializando y profesionalizando, quedando finalmente implantada en las universidades donde, sin embargo, como veremos más adelante, no se admitía a las mujeres en iguales condiciones que a los hombres. Al hilo de estos procedimientos, a los científicos y a su actividad se les otorga, a través de unos principios reguladores de pautas de conducta, una autoridad y una legitimación que facilita su reconocimiento vigoroso en la sociedad. Sin embargo, estas observaciones no deben hacernos olvidar que si bien la ciencia es una actividad humana importante, también es producto de los sujetos que la crean; por tanto, los científicos, consciente o inconscientemente, corren el riesgo de no percatarse de las diversas perspectivas de ésta; con lo que, algunas veces, sería necesario que retrocedieran y

${ }^{1}$ La ciencia, tal como hoy la entendemos, es fundamentalmente una creación de la sociedad occidental. Esto no significa que en otras sociedades, como la oriental, no hayan realizado contribuciones a la ciencia actual, pero después de haber sido filtradas por la ciencia occidental (Solsona, 1997: 7). 
El lado oscuro de la mujer en la investigación científica...

reexaminaran el escenario para corregir o confirmar sus percepciones más próximas. Las ciencias de la naturaleza están muy fragmentadas y especializadas, por lo que hay que evitar que quien investigue sólo perciba una pequeña porción del todo.

Existe un consenso generalizado sobre los beneficios que se han obtenido a partir de la institucionalización de la ciencia, ésta parece condicionar tanto todo el proceso social, que conviene tener cuidado, no vaya a ser que se aleje de sus fines iniciales. Barnes contempla que nuestra cultura está orientada hacia la ciencia con más fuerza que nunca pero, pese a ello, anuncia que nunca ha sido mayor el abismo entre la ciencia y la vida cotidiana; argumentando que su estrecha relación de interdependencia con las instituciones sociales dominantes es la que permite que, a pesar de la especialización y pérdida de otras perspectivas, la ciencia sea una institución estable (Barnes, 1987).

En definitiva, si la función social de la ciencia es, en cierto modo, revelar la realidad, para evitar ocultarla, los científicos deben ser sensibles a las necesidades sociales. Seguir el método científico tal vez no garantice una ausencia de valores, ya que el investigador, al estar inmerso en un determinado contexto social o cultural, de alguna manera, incorpora valores sociales en su orientación científica. Valores de los que debería ser consciente a la hora de investigar. Por tanto, sería entonces idealista pensar que la construcción social de la ciencia está libre de prejuicios y alejada de determinados intereses ${ }^{2}$. Sin embargo, es tal la aprobación de la que disfruta la investigación científica que cualquier otro tipo de indagación alternativa es rechazada automáticamente, impidiendo además que otra información pueda contribuir a ampliar el abanico del saber y se considere científica. Indudablemente el conocimiento presente

${ }^{2}$ E. Lamo de Espinosa, en la conferencia pronunciada en la sesión de clausura del VII Congreso Español de Sociología, organizado en Salamanca (22 de septiembre de 2001) hacía hincapié en que el conocimiento científico-técnico nos rodea de tal modo, es ya parte tan natural de nuestra existencia, que perdemos la dimensión real de su importancia. Para captarla es necesario salir del marco de la actual sociedad del conocimiento, e incluso en alguna medida del marco de la civilización occidental, para vislumbrar su proceso de desarrollo. 
condiciona la manera de asimilar nuevas experiencias, así como la creación de nuevos conocimientos, con lo que se hace necesario y justo dudar de la manera en que se aplica el conocimiento científico, que por otra parte no deja de ser provisional e incierto (Barnes, 1987: 58-59).

No es hasta los años sesenta del siglo XX cuando se empieza a poner en entredicho la concepción que se tenía sobre ciencia. Cabe recordar la alternativa que supuso Kuhn (1962) en lo que a la historia de la ciencia se refiere, desvelando el modo progresivo en que ésta se construye y, mostrando el carácter temporal y tentativo de las teorías que acaban desarmando el mito de la objetividad. Para Kuhn la ciencia es una construcción social y, como tal, inseparable de los procesos que se desarrollan en la sociedad. Está convencido de que las teorías y los paradigmas científicos dependen, por tanto, del contexto histórico, social y económico en el que se crean (Van den Eynde, 1994).

Éste y otros fueron algunos de los motivos que, a partir de la década de los 70 , hacen que necesariamente se amplíe el interés por la epistemología de la ciencia, estimulando que los estudios sociales en torno a ella lleguen a desconfiar de todas y cada una de esas creencias, hasta entonces mitificadas. Pese a todo, los pensadores postkuhnianos elaboran estudios deformados por sesgos androcéntricos, sin considerar el género como herramienta analítica para comprender la historia de la ciencia que es, como veremos, el objeto de las críticas feministas (Harding, 1996).

A partir de esta introducción se trata de exponer que para dar a conocer la realidad es conveniente recuperar la voz de los excluidos de la ciencia, de los que no han tenido opción de intervenir en su construcción, ni han sido destinatarios de la misma. En síntesis, la perspectiva defendida es que ésta se ha construido desde el poder dejando en la sombra a los sometidos en general y a las mujeres en particular. Pues la imagen positivista y racional que se le ha venido atribuyendo a la ciencia coincide con las cualidades que tradicionalmente se han adjudicado al género masculino, frente a la subjetividad e irracionalidad que se ha conferido a lo femenino, en general (Van den Eynde, 1994). 
El lado oscuro de la mujer en la investigación científica...

\section{Situación de la mujer en y ante la ciencia}

Son muchas las explicaciones tradicionales, dadas desde cualquier área, que han servido para justificar la exclusión femenina en el espacio científico (Van den Eynde, 1994). Históricamente, el papel de la mujer ha sido relegado al ámbito privado de la organización social, subestimando su capacidad de investigación. Determinados condicionamientos sociales han limitado sus oportunidades de participación en la ciencia y en otras actividades, dificultando el acceso de éstas a la educación, que prepara para el posterior acceso al ámbito científico.

Durante la Edad Media, el objetivo de la institución educativa consistía en formar para la Iglesia y para la Administración, que eran ámbitos exclusivamente masculinos (Van den Eynde, 1994). La institución universitaria estuvo vedada para las mujeres hasta el siglo XIX y en algunos países hasta el siglo XX. Incluso avanzado ya este último siglo, en el curso 1919-1920, la representación de mujeres en la universidad española era de un $1,5 \%$, porcentaje superado en poco más de siete puntos nada menos que once cursos después (curso 1935-1936) ${ }^{3}$. En cualquier caso, el curso con mayor número de alumnas universitarias en ese intervalo de años fue el curso 1927-1928 en el que el alumnado femenino llegó a representar un 9,2\% (Ballarín, 2001).

En España, a comienzos del pasado siglo, el analfabetismo era algo generalizado que comienza a reducirse cuando las mujeres inician su andadura universitaria. En el contexto originado durante la Segunda República, en nuestro país, se establecen substanciales mecanismos innovadores que promueven la promoción y mejora de la investigación y la educación científica. Si bien el analfabetismo femenino se redujo hasta un $47,5 \%$ durante ese periodo, la brevedad en la vigencia del régimen impide que las reformas establecidas alcancen las aulas universitarias, donde las mujeres sólo representaban un 8,8\% del alumnado. Posteriormente, en un contexto bien

\footnotetext{
${ }^{3}$ Obviamente no debemos olvidar la situación social por la que pasaba nuestro país en esos años.
} 
distinto, el papel que concedía a las mujeres el régimen dictatorial impuesto tras la guerra civil española, limitaba su desarrollo como profesionales e investigadoras. Con todo, entre 1940 y 1969 las mujeres universitarias experimentaron un aumento del 13\% al 30\%, sin embargo, dicho incremento se producía en carreras como Filosofía y Letras, Farmacia, Medicina y Ciencias Sociales, todas ellas consideradas "femeninas", frente a las Ingenierías, donde prácticamente no había ninguna mujer (Pérez Sedeño, 2003).

Parece que con la profesionalización de la ciencia, la prohibición y la expulsión de las mujeres de los universos del saber, en general y, respecto a los conocimientos oficiales, en particular, era lo corriente. Ahora bien, no es lícito obviar que dependiendo del nivel socioeconómico y cultural al que pertenecieran, el grado de prohibición difería. En realidad, la literatura indica que la presencia de mujeres en instituciones científicas no estaba prohibida legalmente, ni ellas estaban desinteresadas por la ciencia, sino que dicha segregación obedecía a la condición de institucionalización, en virtud de la cual las normas institucionales no debían entrar en conflicto con los valores sociales de la época, que en las sociedades del siglo XVI a XVIII eran política e ideológicamente masculinos. De modo que la institucionalización de la ciencia moderna parecía haber legitimado la exclusión femenina de este ámbito y, su papel en dicha actividad era inversamente proporcional al prestigio de la misma (González, 2002).

Concretamente, si centramos nuestra atención en el área de la sociología convencional, hallamos algunas denuncias al desinterés, que desde este ámbito, se ha mostrado por el tema de la mujer (Abbott y Wallace, 1990). No en vano, sintiendo la necesidad de percatarse de la percepción androcéntrica de la sociedad en los clásicos de la sociología, y la ausencia en la teoría sociológica del género, que es fundamental en el tejido social; Durán expone que la presencia de mujeres entre los fundadores de la sociología brilla por su ausencia y, además, que los varones apenas eran conscientes de lo condicionados que estaban por el género al pensar y escribir de manera parcial sobre sus observaciones. Si bien hay que reconocer que, en los tiempos que corren, esta cuestión ha experimentado un cambio tanto en la organización social de la disciplina 
El lado oscuro de la mujer en la investigación científica...

como en la profesión sociológica, de modo que actualmente distinguimos un gran número de mujeres entre los estudiosos de este área (Durán, 1996) ${ }^{4}$.

Tal y como señalaremos más adelante, paulatinamente el enriquecimiento científico ha contribuido a que la cuestión de las mujeres y la ciencia haya pasado de centrarse en el acceso y la igualdad de oportunidades, a hacerlo en el papel del género en su "construcción" (ETAN, 2001). Veremos que Fox Keller (1991) plantea la

${ }^{4}$ En el texto editado por M. A. Durán se expone que:

- Entre los clásicos es Simmel quien más se ocupó de la condición de la mujer, afirmando que la única cultura existente es la de los varones, aún así, no resuelve el dilema de si es posible el nacimiento de una cultura o contracultura femenina autónoma, que no termine obedeciendo y reduciéndose a la cultura masculina dominante.

- Por otro lado, Gil Calvo mantiene la hipótesis o sospecha de que en la enseñanza española de la sociología brilla por su ausencia el análisis de la construcción social del género; dice que el que la propia realidad social ignore a las mujeres relegándolas a esferas selladas para eludir su visibilidad no debe sorprendernos, pero que sí debería hacerlo el que también las ignore el discurso académico sobre la realidad social. El discurso académico no se enfrenta al problema de la constitución del género, sin embargo, tangencialmente, sí se refiere a él, al enfrentarse a otros problemas ajenos pero relacionados. Se oculta la actitud generizada tras los discursos académicos en Sociología.

- A continuación, Jiménez Blanco, explica que la teoría sociológica del siglo XIX, principio de nuestra disciplina, sostiene la primacía de los conceptos colectivos, es decir, los sujetos colectivos dan cuenta de los individuales; las colectividades subsumen a los individuos, cuya singularidad carece de relevancia (varones y mujeres como individuos son partes de esas colectividades, por igual). La primacía de los conceptos colectivos lleva a una teoría sociológica de las macroestructuras (económicas, políticas, culturales...) en las que la presencia de las mujeres era inexistente. Para que la mujer tenga un papel propio en la sociedad tenemos que esperar a que aparezca la antítesis de los conceptos colectivos, la irrupción del individualismo metodológico, que suele asociarse con Max Weber, pero abarca también a otros autores. Cambio de enfoque teórico importante en el descubrimiento de la singularidad de la mujer, con independencia del varón.

- Finalmente, García de León afirma, que el pensamiento sociológico se ha caracterizado desde sus orígenes por ser una respuesta nuevas situaciones sociales... Sin embargo, la literatura sociológica no ha reflexionado sobre cómo son las diferencias sociales entre hombres y mujeres (en el pasado y ahora): sociología androcéntrica, rasgo compartido por todas las disciplinas del saber académico, hasta los Estudios de Mujer, ésta habla de un pasado reciente de la sociología androcéntrica (Durán, 1996). 
posibilidad de crear una ciencia deliberada de género, desde un punto de vista feminista. La ciencia moderna, no es la única práctica para conocer el mundo, sino una forma más de saber, diferente de las demás por tener un método determinado. Fox Keller subraya que: es generalmente aceptado entre los profesionales de la ciencia que la observación es dependiente de la teoría y que la teoría está impregnada de valores, pero al mismo tiempo indica que esta situación coexiste con el convencimiento de que la actividad científica se justifica a sí misma por sus avances y por su capacidad de predicción. Tal y como comprobaremos luego, Harding (1996: 32) va más allá y trata de identificar las tendencias causales de la vida social que dejan huellas de género en todos los aspectos de la empresa científica.

Sin duda, en cuanto al limitado acceso de la mujer al ámbito científico, se distingue como antecedente esencialmente importante la menor proporción de éstas, respecto a la de hombres, dedicadas al estudio y la producción de la ciencia en toda la historia. En cambio, si bien su presencia ha sido inferior a la de los varones, no lo ha sido tanto como se suele afirmar (González, 2002). Para averiguar por qué las mujeres no forman parte de este "coto cerrado" de varones se hizo necesario reformular la historia, esto es, recordar a las mujeres que, injustamente y contra el rigor científico, se habían quedado en el olvido a lo largo del tiempo debido a los sesgos y concepciones tradicionales propios de la misma historia de la ciencia. También hubo que señalar las aportaciones que ellas han hecho a la cultura y a la sociedad, pues han resultado fundamentales para la humanidad ${ }^{5}$.

Algunas estudiosas conscientes de la necesidad de reescribir la historia de las científicas para dar a conocer la relación entre las mujeres y el conocimiento, parten de una posición crítica en relación a la concepción androcéntrica dominante en la historia

\footnotetext{
5 En el siglo IV, Hipatia desarrolló importantes trabajos en álgebra, geometría, matemática y astronomía. Ésta ha sido considerada por siglos la única mujer de ciencia dada la amplia documentación que existe sobre ella por las trágicas circunstancias de su vida y su muerte (http://aupec.univalle.edu.co/informes/marzo98/mujerciencia.html).
} 
El lado oscuro de la mujer en la investigación científica...

de la ciencia. En este sentido concluyen que las contribuciones que las mujeres han hecho en este espacio, han sido filtradas por la mirada de aquellos hombres que han reestablecido la historia. Quienes se han ocupado de indagar en el pasado científico de Occidente, han encontrado que numerosas mujeres habían contribuido a la creación del conocimiento y de la ciencia, muchas más de las que en un principio se podía imaginar (Solsona, 1997: 7-8). Es inevitable entonces, releer con talante crítico y de manera global la historia que hemos recibido, para hacer visibles a las mujeres en todos aquellos ámbitos sociales en que se las ha ignorado y recordar, por tanto, olvidos intencionados.

No obstante, en la Europa continental la imagen clásica femenina de la ciencia permaneció hasta que, en los primeros años de la revolución científica, algunos clásicos la refutaron y la imaginaron como propia de los hombres, rechazando a la mujer culta e ilustrada (Marco, 1999). De manera que hasta no hace mucho tiempo nada se ha sabido sobre Maria Sklodowska que, conocida por Marie Curie al tomar el apellido de su marido, fue la primera persona, hombre o mujer, en ganar el Premio Nobel dos veces, la primera en Física junto con su marido y, la segunda en Química por su trabajo en solitario. Igualmente desconocida ha sido la contribución de Rosalind Franklin, decisiva para la determinación de la estructura helicoidal del ADN, contribución que se apropiaron Wilkins, Watson y Crack, los científicos que recibieron el Premio Nobel por dicho descubrimiento tras la muerte de Rosalind. También se ignoraba la aportación de Mileva Maric a las teorías de Einstein, aún siendo reconocida por el propio físico. Sin más, sirva como ejemplo que entre 1650 y 1710 nada menos que un $14 \%$ de los astrónomos alemanes eran mujeres ${ }^{6}$. Este último dato, como otros muchos, ha sido obviado por la historia de la ciencia (Van den Eynde, 1994).

Efectivamente, la historia de la ciencia, tal y como la conocemos, es una historia parcial en la que las mujeres representan una minoría; una historia que se ha olvidado de aquellas que iban más allá de lo que tradicionalmente se consideraba como

\footnotetext{
${ }^{6}$ Desde el siglo XVI la ciencia moderna se iba configurando en talleres artesanales en los que se practicaba la Astronomía y la Entomología y, las mujeres participaban como aprendices y trabajadoras.
} 
meta apropiada para ellas en el ámbito del conocimiento. Debe entenderse entonces que debemos interpretar la historia de la ciencia como algo más que la historia de unos cuantos hombres que cambiaron drásticamente nuestra concepción del universo. Indudablemente, si mirando hacia atrás en el tiempo nos percatamos de las contribuciones que las mujeres han hecho al conocimiento, proporcionando grandes avances científicos que, sin embargo, se desconocen (Alic, 1991).

Por tanto, al cuestionar las explicaciones generalistas de la ciencia, así como el uso de explicaciones androcéntricas del conocimiento, que autorizaron la ausencia de mujeres como objeto y como sujetos de conocimiento, se pone en evidencia la ausencia de la contribución femenina al discurso histórico tradicional (Ballarín, 2001). Sin embargo, ya bien avanzado el siglo XX, la situación de la ciencia y en concreto de las mujeres científicas cambia radicalmente. La ciencia deja de ser una actividad individual, ejercida con recursos propios y se institucionaliza en las universidades, en la industria y en los centro de investigación estatales. De igual manera, las mujeres van logrando progresivamente igualdad de derechos con los hombres y se las acepta en universidades e instituciones científicas eliminando, al menos, toda discriminación formal (Van den Eynde, 1994).

En cualquier caso, la evidencia empírica muestra que las mujeres todavía permanecen excluidas de los círculos más prestigiosos de la ciencia. Las instituciones de enseñanza y en el Consejo Superior de Investigaciones Científicas (CSIC) en nuestro país son los ámbitos que representan esos círculos en donde las mujeres siguen ocupando los puestos de menor nivel, como veremos con los datos que se ofrecen más adelante.

$\mathrm{Si}$ consultamos las cifras que se ofrecen desde distintas universidades españolas nos damos cuenta de que actualmente la presencia de mujeres entre el alumnado universitario es incluso superior a la de varones. Hace algunas décadas esto parecía injustificadamente imposible, pues éstas no podían acceder al mayor nivel de competencia profesional que proporcionaba la educación superior dado que, además, se les inculcaba que la ciencia estaba más allá de lo que su inteligencia podía alcanzar. 
El lado oscuro de la mujer en la investigación científica...

Desde principios del siglo $\mathrm{XX}$, como consecuencia del continuado progreso social y desarrollo económico que se venía experimentando, la sociedad demandaba una formación más cualificada, incluso para las mujeres. De manera que primero se les reconoce el derecho a la educación, para posteriormente conseguir las titulaciones en el mismo grado y contenido que el varón, al menos formalmente.

En un primer momento parece que el libre acceso sin permiso a la universidad estuvo prohibido para nuestras antepasadas hasta 1910, con lo que ninguna española fue profesora en la misma hasta 1916. Sorprendentemente las academias científicas tardaron aún más en admitir mujeres, no haciéndolo hasta finales de la década de los años ochenta $^{7}$. Es de destacar que actualmente, pese a las variaciones que caracterizan a los sistemas y estructuras de los países europeos, la proporción de las mujeres que ocupan cargos científicos superiores en nuestros días es extremadamente baja, pues en la mayoría de los países, algo menos del 5\% de los miembros de academias científicas son mujeres (ETAN, 2001). A modo de ejemplo recordemos que el porcentaje de mujeres miembros de las academias científicas al inicio de este siglo era del $5.6 \%$ en la Academia Europea o de dos puntos porcentuales menos en la Royal Society de Londres y en la Academia francesa de Ciencias (3.6\%). Estos datos evidencian que la representación de las mujeres en dichos organismos es muy escasa (Instituto de la Mujer, 1999).

En realidad el acceso a la educación en igualdad de condiciones para hombres y para mujeres ha constituido un fenómeno imprescindible para la emancipación de esta última. A su vez, este acceso a las instituciones educativa les ha capacitado para poco a poco ir incorporándose al ámbito científico; de ahí que quede más que justificada la constante reivindicación histórica de acceso de las mujeres.

Actualmente, del análisis de algunos datos disponibles ofrecidos por el

\footnotetext{
${ }^{7}$ A María Cháscales y Margarita Salas, en la Real Academia de Farmacia, en 1987 a la primera y en la de Ciencias Exactas, Físicas y Naturales en 1988 a la segunda.
} 
Instituto Nacional de Estadística ${ }^{8}$ se desprende la idea de que, la participación femenina difiere sensiblemente de unos sectores científicos a otros, concentrándose en los de Enseñanza Superior y Administración pública. Es decir, atendiendo a la clasificación sectorial del personal dedicado a Investigación y Desarrollo se deduce que el mayor porcentaje de investigadoras pertenece a las instituciones privadas sin fines de lucro (52\%), si bien los efectivos de este sector son muy escasos. A continuación se sitúa el sector de la Administración Pública (42.8\%) y el de la Enseñanza Superior (39.3\% de investigadoras). Para el sector de la empresa, esta cifra representa sólo el 19\% del total de investigadores. Parece que más de las tres cuartas partes de las investigadoras se concentran en los sectores de Enseñanza Superior y Administración pública, con lo que aún se constatan importantes diferencias entre las cifras correspondientes a hombres y mujeres en estas actividades, aunque la evolución de la presencia femenina en todos los ámbitos es continuada y de tendencia ascendente.

Desde el CSIC, institución dedicada a la investigación en todas las áreas científicas creada en 1940 en España, se analiza la situación profesional de las mujeres (CSIC, 2003). Situación ésta que parece dinámica pues, las cifras que desde aquí se ofrecen permiten concluir que, en los últimos años la participación de las mujeres en las distintas escalas y especializaciones se ha ido incrementando, aunque todavía entre el personal directivo del organismo sólo se encuentra una mujer por cada tres hombres. Actualmente las mujeres representan aproximadamente el $32 \%$ del total del personal científico, distribuyéndose entre las ochos áreas de investigación en un 37,7\% en Humanidades y Ciencias Sociales, un 30,5\% en Biología y Biomedicina, un $24.4 \%$ en Recursos Naturales, un $39,5 \%$ en Ciencias Agrarias, un $19,6 \%$ en Ciencias y Tecnologías Físicas, un 33,1\% en Ciencia y Tecnología de Materiales, un 44,8\% en Ciencia y Tecnología de los Alimentos y, un 37,2\% en Ciencia y Tecnologías Química. En particular, si nos detenemos en la distribución del personal científico por áreas y

${ }^{8}$ Cifras INE, Boletín Informativo del Instituto Nacional de Estadística, 2003, disponible en http://www.ine.es 
El lado oscuro de la mujer en la investigación científica...

escalas el porcentaje de mujeres en las distintas escalas de las diferentes áreas es inferior al de los varones y, en general, la distribución del personal científico por escalas muestra que el $15,07 \%$ de esas mujeres son profesoras de investigación; mientras que el $30,84 \%$ (casi cinco puntos porcentuales más que en el 2000) son investigadoras científicas, frente al 38,92\% que son científicas titulares, perteneciendo el resto a otras escalas de investigación (CSIC, 2005).

No cabe duda de que aunque algunos mecanismos sigan dificultando el acceso y permanencia de la mujer en la carrera científica, las perspectivas de las que desean seguir una carrera profesional han mejorado. Algunas de las formas más obvias de discriminación de la mujer han desaparecido, se han levantado las barreras que impedían que se licenciaran en ciencias y que fueran miembros de las academias y asociaciones profesionales (Pérez Sedeño, 2003).

\section{Principales orientaciones de las críticas feministas al estudio social de la ciencia}

Siguiendo con el argumento teórico, tal y como iremos vislumbrando en estas páginas, frente a la epistemología tradicional, se dice que no es posible una teoría general del conocimiento que ignore el contexto social del sujeto cognoscente, pues no es posible la abstracción de dicho sujeto con facultades universales. Desde el feminismo se defiende también la idea de que el conocimiento está condicionado por el sujeto y su situación social cultural. La crítica feminista a la ciencia, como aquella actividad que reproduce y legitima la discriminación social de la mujer, surge en los años setenta del siglo XX.

Esta crítica se apoya fundamentalmente sobre dos pilares, por un lado en los movimientos de los años sesenta que desde las Ciencias Sociales propician una visión de la ciencia como producto cultural opuesta a la mitificación y a la visión acrítica anterior; y por otro lado, estriba en los movimientos feministas de la segunda ola, en pleno apogeo en esos años con la acción de las mujeres en el mundo académico, que 
evidencian cómo el carácter hipotético de la ciencia suele ser olvidado por quienes la crean y utilizan (Agudo, 2004).

Ya en los años sesenta del siglo XX, en EEUU ${ }^{9}$ con los Women Studies, empieza a percibirse un desasosiego cada vez mayor por la baja contribución femenina a la ciencia en general y a la ingeniería en particular. Poco después, en 1971 un grupo de mujeres fundó una Asociación de Científicas encargada de luchar por la igualdad y la plena participación de la mujer en la ciencia y la tecnología (ETAN, 2001). Asimismo desde diversos países europeos poco a poco, se va promoviendo la igualdad de oportunidades en estos ámbitos y, entre 1975 y 1985 en la Década de las Mujeres de las Naciones Unidas, comenzaron a surgir recomendaciones específicas con respecto al género, la ciencia y la tecnología (Pérez Sedeño, 2003).

Así es como las feministas comienzan a incorporar una postura clave en el conjunto de las críticas dirigidas a la manera en que se entiende la ciencia, centrando las suyas en desvelar los valores patriarcales que están presentes en esas prácticas (Ortiz, 1999). Estas críticas procuran la reconstrucción de una ciencia no androcéntrica ${ }^{10}$, ni sexista, que incluya al género como categoría de análisis, frente a la ciencia que se sirve de estereotipos de género, socialmente construidos, que propician un discurso que discrimina a las mujeres, conformando el sistema ciencia-género al que se ha referido E. Fox Keller (1991).

De esta manera, la crítica feminista a la ciencia en primer lugar se ocupa de la discriminación de la mujer en la estructura social de la ciencia y de los objetos de estudio de ésta y, posteriormente cuestiona los supuestos del pensamiento occidental

\footnotetext{
${ }^{9}$ Tras un gran trabajo de presión de las científicas y sus aliados, en 1981 el Congreso de los EE UU aprobó una Ley de fomento de la igualdad de oportunidades para las mujeres y las minorías en la ciencia y la tecnología (ETAN, 2001).

${ }^{10}$ Hay que aclarar que cuando hablamos de androcentrismo, nos estamos refiriendo al método científico que está presente en todas las disciplinas de la ciencia, en todas aquellas formas de conocimiento que recurren a la perspectiva de la experiencia social masculina y que influye en todo el proceso científico de elección de metodología y formulación de teoría, identificando lo masculino con lo humano (Ortiz, 1999).
} 
El lado oscuro de la mujer en la investigación científica...

que constituyen la referencia de la ciencia moderna. Al hilo de los diversos cambios sociales acontecidos en la segunda mitad del siglo pasado en España, en los que las mujeres han participado de manera activa, podemos situar el pensamiento feminista de los años ochenta que presenta una posición renovada de la relación entre mujer y ciencia (Aguinaga, 2004).

El feminismo ha intentado terminar con la ausencia de las mujeres en las instituciones científicas y con el predominio del punto de vista masculino generalizado en todos los campos científicos, ofreciendo explicaciones alternativas, aportando teorías y metodologías nuevas.

Como vamos a tratar de mostrar a lo largo de estas líneas, es evidente que las ciencias sociales para poder responder a estas demandas deben buscar una epistemología que incorpore las diferencias sociales, históricas y culturales que existen entre los sexos (Alberdi, 1999). En nuestros días la esencia del debate ha dejado de ser el valor de la verdad o de las prácticas de investigación científica $\mathrm{y}$, se ha orientado hacia la construcción de un saber neutro, sin embargo, parece seguir olvidándose de la existencia de dos sexos y del dominio masculino sobre el lenguaje científico.

\subsection{Diversas posturas en la crítica feminista de la ciencia}

La evolución de las críticas a las que venimos refiriéndonos indica que éstas han extendido la preocupación centrada en la ausencia de mujeres en la ciencia y en las barreras que existían junto a la demanda política de igualdad atendiendo, a su vez, las consecuencias científicas de dicha ausencia y a la falta de rigor científico provocada por influyentes sesgos androcéntricos. Básicamente todas las críticas feministas se dirigen hacia la organización social de la ciencia y comparten su oposición a la práctica científica sexista. En Ciencia y feminismo Sandra Harding (1996) describe el paso de las críticas feministas desde la cuestión de las mujeres en la ciencia, como sujetos y objetos de conocimiento a la cuestión de la ciencia en el feminismo, mostrando que desde 
mediados de los años setenta del siglo XX esas críticas han evolucionado desde una postura reformista a otra revolucionaria.

Concretamente, Harding señala que la cultura occidental es un reflejo de los proyectos sociales de sus fundadores, influida por el género, clase social, raza y cultura de los mismos. Acorde con los comentarios que venimos haciendo, esta filósofa reconoce que el uso social de la ciencia ha cambiado, se ha convertido en un generador de acumulación y, control económico, político y social, con lo que la esperanza de "dominar la naturaleza" para mejorar la especie se ha convertido en el esfuerzo para conseguir un acceso desigual a los recursos naturales para fines de dominación social.

Añade que las críticas feministas cuestionan la división de trabajo por género en éste ámbito, pues cree que la diferencia de género es el origen más antiguo, universal y poderoso de muchas conceptualizaciones (1996: 15-16). Para argumentar la opinión anterior, Harding expone que la vida social "generizada" se produce al asignar metáforas dualistas de género a diversas dicotomías percibidas que no obligatoriamente tienen que ver con las diferencias de sexo (simbolismo de género) para organizar la actividad social (estructura de género) que es una forma de identidad individual, socialmente construida (género individual). Esta exposición la hace con la intención de reprochar que algunas de las críticas feministas de la ciencia no reconozcan las relaciones entre lo que ella entiende por género simbólico, división de trabajo según género y género individual, concluyendo que con este tipo de críticas no se puede eliminar el androcentrismo en la búsqueda del conocimiento.

En cualquier caso, esta autora, sostiene que una ciencia crítica y reflexiva debe ser el modelo de las demás, y que la búsqueda del saber ha de ser más integradora de lo humano: Sin embargo, declara que las críticas feministas que afirman que también la ciencia está generalizada parecen amenazadoras para el orden social, pues para intentar terminar con esa mirada sesgada alternan, dice, varios proyectos ${ }^{11}$ que se contraponen

\footnotetext{
${ }^{11}$ Cinco programas de investigación colmados según Harding de carencias teóricas:
} 
El lado oscuro de la mujer en la investigación científica...

entre sí, aunque sean plenamente conscientes de la carga androcéntrica de la actividad científica (Harding, 1996).

No cabe la menor duda de que el feminismo es un movimiento político que tiende al cambio social, Harding consciente de ello reconoce la situación paradójica a través de la cual no se puede incrementar la objetividad de una investigación con una indagación politizada. Ante estas circunstancias cuestiona los supuestos científicos que originan las que describe como investigaciones cargadas de sesgos androcéntricos, poniendo en cuestión las respuestas que desde el feminismo se han dado para enfrentar dichos supuestos.

La primera respuesta que Harding cuestiona es la de el empirismo feminista, señala que se trata de un enfoque que ocasiona el problema de la "mala ciencia" al especular que los sesgos sociales del sexismo y el androcentrismo se pueden erradicar a través de la ferviente adhesión a las normas metodológicas de la investigación científica. Este primer cuestionamiento lo justifica indicando que esta solución, aún

1. Los estudios de equidad donde las mujeres llegan a ser como los hombres, suponen una meta baja limitada a la simple igualdad.

2. Los estudios sobre los usos y abusos de la biología, las ciencias sociales y sus tecnologías han revelado de qué forma se utiliza la ciencia al servicio de proyectos sociales sexistas, racistas, homofóbicos y clasistas, políticas reproductivas opresoras.

3. Las críticas a los usos sexistas de la ciencia parten de dos supuestos problemáticos: la existencia de una investigación científica pura, independiente de valores y la existencia de usos adecuados de la ciencia que pueden confrontarse con los inadecuados.

4. Las críticas de la biología y las ciencias sociales, la selección y definición de problemas hacia la percepción de los hombres. La "mala ciencia" asume que los problemas de los hombres son los de todo el mundo. Imposibilidad de distinguir entre mala ciencia y la ciencia al uso, ¿puede haber investigaciones independientes de los valores?

5. La preocupación por mantener dicotomías rígidas en la ciencia son deseos específicamente masculinos, occidentales y burgueses, el progreso humano exige que el primero consiga la dominación sobre el segundo, pero no podemos imaginar una forma científica de búsqueda del saber que prescindiese de la distinción entre objetividad y subjetividad, entre razón y emociones.

Además hay diversas investigaciones, según la autora, epistemológicas que han sentado las bases de una forma alternativa de entender, de saber, estas epistemologías feministas están en conflicto, ésta es la cuestión principal de Ciencia y feminismo. 
siendo atractiva, no deja de perturbar el propio empirismo, pues la identidad social del investigador no es irrelevante y, lamentablemente las creencias empiristas todavía están presentes en la mayoría de las críticas feministas de la ciencia, lo que impide teorizar adecuadamente sobre el género.

A continuación orienta su crítica hacia el enfoque del punto de vista feminista, fundamentado en el reconocimiento de que la experiencia social de las mujeres proporciona el único punto de partida para descubrir el sesgo masculino de la ciencia. Esta perspectiva iría en la línea defendida por Fox Keller, quien defiende que la posición dominante de los hombres en la vida social se traduce en un conocimiento parcial y perverso, mientras que la posición subyugada de las mujeres abre la posibilidad de un conocimiento más complejo (Harding, 1996, 24). Sin embargo, Harding critica a los nuevos estudios sociales de la ciencia por no haber sido lo bastante críticos y no haber cuestionado los supuestos a partir de los que los cánones de investigación y las prácticas de la ciencia son independientes del género. En general expone que la mayoría de estos estudios, como sus antecesores de orientación empirista, son renuentes a considerar los efectos de las identidades y conductas de género, las disposiciones institucionales de género y el simbolismo de género sobre la ciencia (Harding, 1996: 174).

De los comentarios anteriores se infiere que indudablemente la ciencia, como institución dominante, invade todos los aspectos de la vida moderna y tiene capacidad para influir en cualquier área de la sociedad actual. Sin embargo, las críticas que han surgido respecto a esas influencias a veces se han olvidado de que la ciencia, a su vez, es el resultado de una interpretación unilateral y masculina de la realidad. Una evidencia de este olvido la encontramos en la obra de Woolgar (1992) quien en primer lugar plantea, no sin cautela, que el estudio social de la ciencia se presenta como una nueva perspectiva que pretende dar una explicación diferente. No obstante, dicho autor advierte de la imposibilidad de tratar la ciencia como un objeto de estudio diferente, así como de la dificultad que supone la separación del objeto de estudio al hacer cualquier 
El lado oscuro de la mujer en la investigación científica...

tipo de análisis; pues la propia ciencia es producto de una construcción social, la observación no es neutral y, está subyugada a un contexto social determinado.

En definitiva, Woolgar, para afrontar las carencias de los estudios sociales de la ciencia, así como las de las demás tradiciones, propone un punto de vista alternativo, más reflexivo en su estudio, con la inversión y retroalimentación como métodos de estudio, criticando igualmente la unidireccionalidad metodológica. De esta manera, expone cómo la Sociología de la Ciencia trataba a esta institución como una caja negra que no intentaba abrir, concluyendo que es la Sociología del Conocimiento la que intenta abrir esa caja para conocer la construcción del conocimiento científico.

En general los estudios sociales de la ciencia se han olvidado del rol de género, con lo que, más allá de estos planteamientos, Fox Keller (1991: 11), convencida de que la ciencia está arraigada en principios androcéntricos, se pregunta en qué medida la naturaleza de la ciencia está ligada a la idea de masculinidad y qué significaría la ciencia si fuera de otra forma distinta. En Reflexiones sobre género y ciencia, Fox Keller expone como la teoría feminista, con la intención de remediar la ausencia de mujeres en la historia del pensamiento social y político, junto con los estudios sociales de la ciencia, hacen que nuestros planteamientos sobre la relación entre ciencia y sociedad se modifiquen en el caso de los segundos. La autora reflexiona sobre cómo la conjunción de ambas perspectivas, la perspectiva de los estudios sociales de la ciencia y la de la teoría feminista, ha permitido identificar el papel crítico que tiene la ideología de género al mediar entre la ciencia y las estructuras sociales. La conjunción de ambos enfoques permite hacer una crítica radical de las disciplinas tradicionales al demandar que se examinen los supuestos fundamentales, en cierta manera, mitológicos que aún prevalecen en las ciencias y, que ponen a la objetividad y a la razón en el lado masculino y a la subjetividad, el sentimiento y la naturaleza en el lado femenino. Por tanto, concluye que si bien los estudios sociales de la ciencia rechazan la "neutralidad científica", se olvidan de que la ciencia ha sido producida por un subconjunto de hombres blancos de clase media y ha evolucionado sobre un ideal de masculinidad 
particular, al escapárseles la influencia de algunos determinantes extracientíficos en el desarrollo del conocimiento científico ${ }^{12}$.

Sin embargo, la manera que Fox Keller (1991) encuentra de paliar esas omisiones es a través de la perspectiva feminista que, a su modo de ver, proporciona un tema y un método de análisis para investigarlo. La idea central de este texto es que la ideología de la objetividad científica traiciona sus propios propósitos y sólo puede percibirse a través de las lentes de la diferencia, es decir, dando un salto fuera de la comunidad científica masculina ${ }^{13}$. Es por ello que deduce que si hubiera más mujeres dedicadas a la ciencia, podría surgir una ciencia diferente. Defiende que una ciencia practicada por mujeres proporcionaría una mejor y más compleja imagen del mundo, ahora bien, se le escapa que esta idea tropieza con la visión formal de ciencia determinada por su metodología lógica y empírica, motivo por el que posteriormente será criticada, como veremos a continuación.

Harding, frente al enfoque del punto de vista feminista presentado más arriba, argumenta que la experiencia social de las mujeres no es la misma para todas, sino que difiere debido a que éstas pertenecen a clases sociales, razas y culturas diferentes. Posteriormente esta argumentación la lleva a lo que se conoce como escepticismo postmodernista, pues no cree que la objetividad se pueda lograr mediante la neutralización de valores, sino más bien comprometiéndose con ellos, especialmente con los valores participativos y emancipatorios. Es por ello que el último ataque de las críticas feministas de Harding se dirige hacia el enfoque del postmodernismo feminista,

\footnotetext{
${ }^{12}$ Determinantes extracientíficos como la psyche humana individual, dice E. Fox Keller, pues la ciencia es una actividad personal así como social.

${ }^{13}$ Fox Keller (1991) parece convencida de que ha sido esta postura, la del feminismo, la que ha permitido que ella pudiera explorar el estatus que tenía de mujer científica, puesto que la intimidad que ha mantenido con los objetos que estudia la ha proporcionado un manantial de su capacidad como científica. En definitiva, para mostrar que ciertos problemas en la ciencia han de observarse de manera holística, presenta la biografía de Bárbara Mc. Clintock, (analiza su método de investigación, genetista) y concluye que si no hubiera investigado una mujer desde su punto de vista o perspectiva no se hubieran obtenido importantes resultados.
} 
El lado oscuro de la mujer en la investigación científica...

que discute los supuestos científicos fundamentales. Esta postura se presenta en oposición a los supuestos de las dos que le anteceden, desconfiando de los enunciados universales y, se inclina por la utilización de principios para investigar la diversidad, lo que en opinión de Harding origina sus propias tensiones, sin embargo ofrece riqueza conceptual para poder indagar más allá del ámbito limitado del investigador.

En último término, es justo reconocer que Harding, aunque reprocha estas críticas, sostiene que todas y cada una de ellas son necesarias para hacernos ver que los discursos tradicionales dominantes están repletos de incongruencias socialmente dañinas, por lo que es necesario buscar una ciencia informada por una moral y una política emancipadoras, una ciencia con fines emancipadores -con valores participativos, antirracistas, anticlasistas y antisexistas- a pesar de estar inmersa en un entramado occidental, masculino y burgués (1996). Es a los valores coercitivos a los que culpa de ser causantes del deterioro de la objetividad; frente a los valores participativos que disminuyen las deformaciones y mistificaciones de las explicaciones e ideas de nuestra cultura.

En realidad, para esta autora una ciencia objetiva ha de incluir un examen autoconsciente y crítico de las relaciones entre la experiencia social de sus creadores y los tipos de estructuras cognitivas promovidas en su investigación, a través de lo que se dará sentido también a la experiencia social de las mujeres. Por tanto afirma que la crítica feminista en la búsqueda de una ciencia no sexista debería asumir la imposibilidad de investigación, en el sentido de ausencia de valores e intereses sociales en el proceso de investigación, matizando que no es buena ciencia la que está libre de valores, sino la que incorpora los buenos valores (1996).

\subsubsection{Una nueva problemática medioambiental}

Las reflexiones anteriores se encuentran a su vez con una nueva problemática, ahora de una orientación ecológica y ambiental, sobre la que el pensamiento feminista vuelve a tener gran influencia al reafirmar su crítica a la "ciencia moderna" que parece 
dominar sin límites sobre la naturaleza, de manera que dicho dominio no se consideraba dentro de los objetivos propios de la actividad científica. Concretamente, desde esta nueva perspectiva, se hace una llamada de atención sobre los problemas ambientales con los que convivimos. Barnes (1987: 4) ya advirtió sobre la cautela que hemos de tener frente al progreso de la ciencia, progreso que desborda el desarrollo de los recursos e infraestructura necesarios para soportarla y sostenerla. De igual manera E. Fox Keller (1991) reconocía que recientemente se había prestado mucha atención a los abusos tecnológicos de la ciencia moderna y que, en las discusiones que surgían respecto a este tema, se culpaba de las distorsiones al programa científico, intrínsecas a su ambición de dominar la naturaleza sin ofrecer una explicación adecuada.

Al hilo de este enfoque, lo que se conoce como ecofeminismo defiende que las mujeres están muy cerca de la naturaleza, rechazando la tecnología "masculina" basada en la dominación de lo natural; en este sentido, algunas autoras han destacado el carácter inherentemente patriarcal de la tecnología occidental (González, 2002). El ecofeminismo se presenta como una tendencia orientada a la búsqueda de una "nueva ciencia feminista" que esté abierta al libre intercambio de ideas más democráticas y cooperativas, comprometidas con la conservación del medio ambiente e, interesadas en la explicación y el entendimiento de los procesos naturales más que en el descubrimiento y el dominio de los hechos.

Es dentro de esta corriente donde podemos encajar la contribución que hace, desde una perspectiva holística y no dualista ${ }^{14}$, Valdana Shiva a la modificación en la orientación de las primeras generaciones feministas. Esta teórica se inclina por una concepción amplia de la ecología en la que encuentran apoyo las luchas de los grupos marginados contra la desigualdad, explotación y represión. De igual manera, nos alerta contra los peligros que suponen la apropiación, por el poder -gobierno y élites- del vocabulario y las metáforas relativos al medio ambiente. En definitiva, con el objetivo de profundizar sobre esta problemática, Shiva (1995) pretende buscar una alternativa al

\footnotetext{
${ }^{14}$ Véase prólogo de Rajni Kothari, a la obra de Valdana Shiva (1995).
} 
El lado oscuro de la mujer en la investigación científica...

paradigma científico dominante que define como esencialmente machista, procurando poner en relación los objetivos que vienen persiguiendo los movimientos ecológico y feminista, con la intención de llegar más allá de lo que lo han hecho éstos, y darles un mayor rigor científico.

La autora orienta su preocupación hacia la búsqueda de alternativas a la ciencia y tecnología dominantes, así como al paradigma del desarrollo reduccionista enfocado sólo a unos cuantos y no a todos. No obstante, es justo reconocer que el dualismo naturaleza y cultura hace que se cuestione la corriente del ecofeminismo, ya que no deja de haber posiciones enfrentadas en lo que respecta a la relación de las mujeres con la naturaleza. Asimismo, hay quien piensa que el feminismo se enfrenta a las instituciones patriarcales y a los valores sociales y culturales que devalúan y marginan a las mujeres, pero pasa por alto la devaluación que dichas instituciones generan sobre la naturaleza.

Pronto empezamos a comprobar que entre las críticas que se hacen a Shiva están las que se refieren a la equivalencia que hace en su obra, explícita e implícitamente, entre mujer y naturaleza, suponiendo a todas las mujeres partidarias de la protección del medio ambiente, defensoras de la vida y procuradoras de equidad (Shiva, 1995). Aunque se le reconoce consciente del problema, ella no hace una distinción suficiente entre los diversos tipos de mujeres.

Fundamentalmente, esta feminista indaga sobre el lugar que tienen las mujeres del Tercer Mundo en el medio ambiente, mujeres que define a la vez como salvadoras y víctimas de lo que viene a llamar "el mal desarrollo". Dicha exploración la lleva al convencimiento de la existencia de una estrecha relación entre la degradación de la naturaleza y la de la mujer en la sociedad contemporáneas, degradaciones derivadas, argumenta, del mal desarrollo explotador o desarrollo económico, y de las actividades humanas que se basan en dicho proceso ${ }^{15}$. Ante esta situación, piensa que la única alternativa para la supervivencia y liberación de la naturaleza, de la mujer y del hombre es el ecologismo basado en el equilibrio y la diversidad, lejos del dominio científico

\footnotetext{
${ }^{15}$ http://www.redtercermundo.org.uy (07/01/04)
} 
moderno y patriarcal, de la explotación y del mal desarrollo capitalista que originan la destrucción de la vida.

Definitivamente, la autora señala que recuperar el principio femenino supone un desafío intelectual y político al proyecto patriarcal de dominación y destrucción originado por el mal desarrollo justificado intelectualmente por las categorías reduccionistas del pensamiento masculino y patriarcal y, la acción científica que entraña la subyugación de la naturaleza y de la mujer.

Para consolidar esta aserción, Shiva nos refresca la memoria mencionando que se refiere al proyecto denominado por Harding (1996) “occidental, burgués y masculino" y por E. Fox Keller (1991) producto científico de un "subconjunto particular de la raza humana -es decir, casi enteramente por hombres blancos de clase media". Los padres fundadores de la ciencia moderna, señalaba esta última, dependían explícitamente del lenguaje de los géneros; pues ellos buscaron una filosofía que mereciera llamarse "masculina", que pudiera distinguirse de sus ineficaces predecesoras por sus poderes "viriles", y "su capacidad de poner a la naturaleza al servicio del hombre y hacerla su esclava" (Fox Keller, 1991: 15).

\section{Luces de la mujer en el ámbito científico: avances y alternativas}

A grandes rasgos, de lo anterior deducimos que la actividad científica debe analizar la realidad alejándose de generalizaciones y verdades parciales, sin renunciar a teorizaciones holísticas, todo ello desde la perspectiva de género, para conseguir deslegitimar la discriminación sexual de la que venimos siendo víctimas en todos los medios. Como decíamos más arriba, fue a partir de los años sesenta y setenta del siglo $\mathrm{XX}$ con la segunda ola del movimiento feminista, cuando ven la luz los procesos de reflexividad científica de los Estudios de Mujer, en un primer momento en el mundo anglosajón, y posteriormente en Europa y España (Agudo, 2003).

Esta línea de investigación científica, necesaria para incrementar el conocimiento de nuestra sociedad, merece tomar la importancia de todas las demás, 
El lado oscuro de la mujer en la investigación científica...

pues se encarga de hacer visible a la "otra" mitad de la sociedad que ha permanecido ausente en el ámbito científico. Estos estudios con sus aportaciones a la organización social de la ciencia, revelan cómo participan en ella las mujeres, manifestando el papel que juegan algunos símbolos y metáforas androcéntricas en su construcción. En definitiva, los estudios sobre género y ciencia, aún caracterizándose por su diversidad de posturas y concepciones, comparten la oposición al androcentrismo científico. No obstante, más que limitarse a la mera función crítica, estos análisis, se enfrentan a su vez a la difícil tarea de proponer alternativas constructivas para estructuras y contenidos de conocimiento científico insatisfactorios en las que se reconozca la ciencia como una práctica social (González, 2002).

De lo anterior, nos debe haber quedado claro que la crítica feminista de la ciencia se inspira en la baja presencia de la mujer en este ámbito, así como en el vacío existente de los contenidos científicos desarrollados por mujeres. Fue esta situación, como hemos constatado, la que provocó el estudio de dichas contingencias de manera interdisciplinar incitando, además, a abordar las diferencias entre hombres y mujeres tanto en la universidad, que prepara el acceso al "mercado de la ciencia", como en cualquier ámbito social, para terminar con una realidad que se presenta diferente para las mujeres y para los hombres.

Los Estudios de Mujer, que van institucionalizándose en las universidades a partir de esa época, con la intención de desmoronar aquel espacio cargado de tradición masculina, pretenden reformar las disciplinas, incorporando nuevos planteamientos epistemológicos a todos los ámbitos científicos. Estos estudios parten del reconocimiento de la carga sexuada del saber, intentando aplicar la perspectiva de género a todos los objetos de conocimiento (Ballarín, 2001). Todo ello para que sea posible concebir a las mujeres dentro de la ciencia y reconocerles espacios nuevos lejos del ámbito doméstico habitual.

En cierto sentido, lo que entendemos por conocimiento se ha ido construyendo con metáforas, valores y símbolos encargados de representar y reproducir las relaciones jerárquicas entre hombres y mujeres. Ahora bien, la perspectiva de género propone la 
construcción de un conocimiento situado en un contexto histórico, localizado y crítico frente a las aspiraciones universales que ocultan procesos de exclusión, luego el concepto de género, constituyendo una categoría de análisis científico, ofrece una nueva perspectiva metodológica, así como una nueva herramienta para el análisis ${ }^{16}$.

Si bien las teorizaciones sociales no incorporaron la categoría analítica del género hasta finales del pasado siglo $\mathrm{XX}$, recientemente esta perspectiva ha conseguido introducir nuevos objetos de estudio relacionados con intereses y experiencias de las mujeres, nuevos puntos de vista y de partida que proporcionan conocimientos diferentes. Aún así, consciente de esta problemática, Harding cree que el uso del género como categoría analítica relevante en las ciencias naturales, experimentales y tecnológicas es difícil porque la propia estructura metodológica -de estas cienciascarece de una tradición autocrítica e interpretativa de sus propios métodos, además, estas disciplinas han convertido en mitos los presupuestos básicos de objetividad, neutralidad y racionalidad (Ortiz, 1999).

\section{1. ¿Qué dicen los datos acerca de estas teorías?}

Ahora bien, si nos movemos de la teoría a la práctica, no debemos dejar en el tintero las aportaciones que se han venido haciendo, incluso desde la Unión Europea, para reconocer el lugar que se les debe otorgar a esa mitad de la población que hasta ahora se había tendido a olvidar. El interés europeo por el tema de la mujer y la ciencia se justifica, particularmente, por la escasa representación de las mujeres en las carreras científicas.

\footnotetext{
${ }^{16}$ La literatura científica ha ido simplificando el uso de este concepto, pero utilizarlo como categoría de análisis supone una renovación epistemológica interesante en la investigación social. Según Scott (1990: 25) "género" fue un término propuesto por quienes afirmaban que el saber de las mujeres transformaría los paradigmas de las disciplinas. Las estudiosas feministas ya indicaron que estos estudios alumbrarían temas nuevos y harían necesario reconsiderar de manera crítica las premisas y normas de la obra académica presente.
} 
El lado oscuro de la mujer en la investigación científica...

La década de los años setenta ${ }^{17}$ fue fundamental en nuestro país para dar un giro en las características que hasta entonces habían distinguido al sistema universitario. Según datos del Ministerio de Educación y Ciencia, en España, no fue hasta mediados de la década de los ochenta (en el curso 1986-87) que las universitarias superaron por primera vez el $50 \%$ del total del alumnado, pero este aumento se orientó hacia aquellas especialidades en las que ya existía mayor porcentaje de mujeres como Farmacia o Psicología; concretamente el 60\% de estas alumnas se concentraban en las Facultades de Filosofía y Letras y Derecho. Otras opciones científicas como Ingenierías y Arquitectura concentraban una cantidad menor de mujeres, pues estas titulaciones, sólo eran elegidas por el $1,8 \%$ de las universitarias ${ }^{18}$. Sin embargo, desde los primeros años de esta década se aprecia un incremento de matriculadas prácticamente en todas las área, esto es, el $47 \%$ de los estudiantes de ciencias experimentales, es decir casi la mitad, eran mujeres. En Ciencias de la Salud lo eran el 49,9\%, mientras que en las Escuelas Técnicas Superiores, las féminas representaban casi treinta y ocho puntos porcentuales menos que en el área anterior. Un alto porcentaje de mujeres era también el que se matriculaba en Ciencias Jurídicas y Sociales (44,5\% del total) y en Humanidades $(63,5 \%$ del total eran mujeres). Ahora bien, años después, las mujeres son ya mayoría en la universidad española, constituyen más de la mitad del alumnado universitario, además son mayoría en todas las áreas, salvo en las Escuelas Técnicas Superiores, donde sólo el $20,6 \%$ de los matriculados son mujeres y, en Ciencias Experimentales, que las mujeres representan un 48,1\% del total. Actualmente, la presencia de mujeres en las aulas universitarias ha aumentado hasta casi alcanzar la de los hombres en todas las áreas, no obstante, éstas continúan siendo mayoría en de Humanidades, pero no en Ingenierías (Pérez Sedeño, 2003).

${ }^{17}$ En 1970 se aprobaba en España la Ley General de Educación, por la que niños y niñas se educaban juntos y, posteriormente, en 1977 se aprobaba la Constitución según la cual ninguna persona puede ser discriminada por razón de sexo.

${ }^{18} \mathrm{En}$ : http://www.campus-oei.org/oeivirt/rie06a03.htm 
Sin duda, en todos los países europeos las mujeres están insuficientemente representadas en los escalones superiores del profesorado y de investigadores, es por ello y por la escasa atención que en Europa, desde los primeros Programas Marco, se había prestado a las cuestiones de género en las políticas de investigación, que desde 1999 existe un subprograma ubicado en Ciencia y Sociedad del Quinto Programa Marco de Investigación: "Mujer y Ciencia", que exige tomar en cuenta la política comunitaria de la igualdad de oportunidades. Pese al embarazoso pronóstico que podemos tener de la situación de las mujeres en Europa debido a la ausencia de datos fiables, accesibles y organizados, desglosado por sexo y niveles, indiscutiblemente es más fácil encontrar científicas entre las disciplinas de Ciencias Sociales y Biológicas, que entre las de Química, Física o Ingeniería, más colmadas de varones.

Si bien la disminución de la representación femenina a medida que aumenta el rango sigue el mismo patrón en todas las áreas, parece entonces que las disciplinas con menor número de mujeres, en la mayoría de los países, suelen ser las más valoradas. En la actualidad, en estos países el número de licenciadas tiende a ser superior al de los licenciados, sin embargo, el mercado laboral científico es aún un universo masculino, pues las mujeres tienen una representación menor en las ciencias y en los órganos encargados de la toma de decisiones en áreas científicas, pues ocupan sólo un $10 \%$ de los puestos directivos de las instituciones docentes y un porcentaje aún menor en las empresas $^{19}$. Sin ir más lejos, el porcentaje de catedráticas en los países europeos es muy bajo y escila entre el 5\% de los Países Bajos y el 18\% de Finlandia, pasando por un porcentaje intermedio en el caso de las catedráticas españolas. La evolución de estos porcentajes en varios países a lo largo del tiempo se caracteriza por cierta estabilidad durante los años 80, empezando a experimentar un aumento lento pero constante a principio de los años 90.

Es cierto también que las mujeres abandonan la academia a un ritmo superior al de los hombres, aún así, actualmente la proporción de hombres y de mujeres entre los

${ }^{19}$ Ver: http://europa.eu.int/comm/research/scie.../women-science_es.htm 
El lado oscuro de la mujer en la investigación científica...

estudiantes de licenciatura es bastante similar en varios países: Bélgica, Francia, Alemania, Países Bajos, España y Reino Unido, generalmente hay más mujeres universitarias que varones, se licencian más y además obtienen mejores resultados, sin embargo, la proporción de mujeres cae en picado en los estudios postdoctorales, con los que normalmente empieza a forjarse una trayectoria profesional (ETAN, 2001). Con todo, si bien los estudios de doctorado son el primer escalón en la carrera docente e investigadora $\mathrm{y}$, a principios de la década actual las mujeres doctorándose suponían el $50.84 \%^{20}$, superando por poco a sus compañeros varones, la diferente distribución por áreas se mantiene, además el porcentaje de doctoras es de casi catorce puntos porcentuales inferior al de doctores (Pérez Sedeño, 2003).

Sin lugar a dudas, si se consiguiera alcanzar una participación equitativa y total de las mujeres en todos los niveles se podría mejorar la diversidad y estimular nuevos avances en la ciencia europea. Con el Sexto Programa Marco (2002-06) el Grupo de Helsinki integra la dimensión de género para facilitar la andadura a las mujeres de ciencia, no sólo hacia la igualdad de oportunidades, sino también hacia la igualdad de resultados. Dicha andadura, en la que actualmente nos encontramos, debe suscitarse a través de un objetivo transversal en todos los sectores de intervención, para mejorar la utilización de recursos humanos y progresar en la investigación desde nuevas perspectivas. Por tanto, para promover la participación de las mujeres en la investigación científica, la Comisión Europea está tomando medidas para garantizar una transversalidad de género y, facilitar que las mujeres avancen en la actividad científica a través, por ejemplo de la creación de redes de mujeres en este espacio.

Por tanto, encontramos actuaciones políticas importantes ante la presencia no

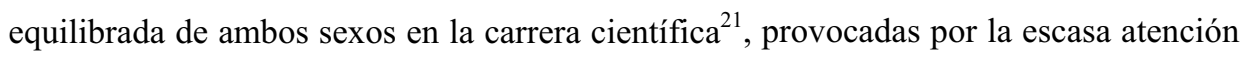
que se ha prestado a las cuestiones de género en las políticas de investigación

\footnotetext{
${ }^{20}$ Estadística de Enseñanza Universitaria, Instituto Nacional de Estadística.

${ }^{21}$ Véase todo lo referido a: Grupo de Helsinki sobre mujer y ciencia. Las políticas nacionales sobre mujeres y ciencia en Europa.
} 
europeas $^{22}$, que lleva a reflexionar sobre la política comunitaria de la igualdad de oportunidades. Alertando sobre la infrarrepresentación femenina en escalas superiores del profesorado y de la investigación, dichas políticas integran la dimensión de género para facilitar el camino a las mujeres de ciencia. En definitiva, promover la igualdad de manera transversal debe ser un objetivo en todos los sectores de intervención para mejorar la utilización de recursos humanos y progresar en la investigación a partir de nuevas perspectivas. Es por ello, que la colaboración de las organizaciones europeas para facilitar el avance de las mujeres en la actividad científica, puede resultar una medida favorable que promueva a su vez la creación de redes y de vínculos entre ciencia y sociedad, incluyendo de algún modo la presencia de las mujeres en estas áreas.

\section{A modo de conclusión}

Parece claro que no resulta nada ventajoso ceder ante el desequilibrio entre investigadores e investigadoras. Sin embargo, la ciencia continúa siendo propiedad masculina a pesar de la creciente presencia de las mujeres en este ámbito, lo que no deja de constituir un elemento novedoso en las últimas décadas frente a aquellos tiempos en que había sido un espacio vedado para ellas (Fernández, 2000).

Como consecuencia de las transformaciones sociales y culturales acontecidas en las últimas décadas, las mujeres tienen más posibilidades de dedicarse a la ciencia y a la investigación. A nivel teórico nadie duda de la igualdad en la universidad, pero viejos tópicos sexistas persisten, haciendo que se desatienda la igualdad de resultados (Camps, 1998). Sorprendentemente, el número de licenciadas es superior al de licenciados, pero en el "mercado de la ciencia", la representación resulta ser masculina. Estas circunstancias son el producto de la persistencia de lo que metafóricamente se ha venido

${ }^{22}$ Véase: Informe del Grupo de Trabajo de ETAN sobre las mujeres y la ciencia: Política científica de la Unión Europea: Promover la excelencia mediante la integración de la igualdad entre géneros, disponible en:

http://www.cordis.lu/rtd2002/science-society/women.htp

Cuestiones de género, 2006, $\mathrm{n}^{\circ} 1$, pp. 15/51 
El lado oscuro de la mujer en la investigación científica...

a denominar el "techo de cristal" que mantiene a las mujeres alejadas de los puestos de mayor prestigio, poder y responsabilidad científica.

Si bien cada vez son más las mujeres que acceden a la universidad, tanto por la creciente importancia concedida en nuestra sociedad a los títulos universitarios como por los cambios ideológicos relacionados con las expectativas del rol social de la mujer, resulta que siguen teniendo opciones educativas y profesionales diferentes. La opción de elegir una disciplina $u$ otra, parece estar determinada por una serie de condicionantes sociales: prejuicios, estereotipos y expectativas sobre lo que tradicionalmente se ha considerado que corresponde atribuir a hombres y a mujeres. La selección por especialidades sigue siendo un elemento discriminatorio, las mujeres suelen elegir las conocidas como "ciencias blandas" y calificadas como femeninas (Humanidades y Ciencias Sociales) y los hombres tienden a ubicarse en las "ciencias duras". Por tanto, la elección de estudios universitarios que hacen las mujeres continúa estando, en cierto modo, supeditada a un proceso de socialización cargado de género.

Estas orientaciones evidencian que las aspiraciones profesionales de las féminas suelen ser más limitadas que las de los varones. Ahora bien, la participación de las jóvenes en los estudios superiores científicos y técnicos supera con creces a las del pasado, las opciones femeninas están cambiando. Cada vez menos se puede hablar de la existencia de "estudios masculinos o femeninos". Indudablemente se va rompiendo la rígida y tradicional división en ramas de estudios.

En cambio, a pesar de su prometedora presencia en facultades científicas, cuando analizamos la trayectoria profesional, esta tendencia se invierte. Pues, cuanto más alta es la jerarquía, menos son las mujeres. De ahí que exista un reducido número de mujeres en los puestos directivos de las universidades europeas, representando una minoría en los debates en los que se toman decisiones. Podemos citar, en particular, el caso de España: donde en 1999 nada más que el 1,6\% de los rectores y el 9,8\% de los vicerrectores eran mujeres (ETAN, 2001).

Una vez más, diversas estadísticas analizadas sobre educación y mercado laboral, muestran un considerable incremento del número de mujeres que componen la 
plantilla del profesorado universitario, sin embargo se detectan pautas que nos indican que se trata de una presencia localizada en algunas áreas del conocimiento en general y, en determinadas categorías profesionales en particular. Es incuestionable la tendencia a la evolución de las mujeres en el colectivo de profesores, sin embargo su presencia disminuye en la categoría académica superior: la cátedra donde las mujeres representan un 12,41\% frente al 57,54\% de catedráticos (García de Cortázar y García de León, 1997). Señálese, por ejemplo, que el porcentaje de participación femenina en las labores docentes de la universidad se sitúa en torno al 33\%, porcentaje que duplica al de la participación femenina docente de finales de los años setenta ${ }^{23}$. En 1977-78 el 17.9\% de la que la mayoría la representan la categoría de profesor ayudante $(48.84 \%)$, seguido de la de asociadas $(34,91 \%)$ y de la de titulares (34,01\%) en el curso 2000-01. De igual manera la distribución del profesorado femenino en las universidades españolas por ramas es la siguiente: en Humanidades representan un $16.5 \%$, mientras que en las Ciencias Jurídicas. Sociales son el 34\%, en Ciencias Experimentales el 11.7\%, en Ciencias de la Salud el 25.2\% y en las Técnicas el 12.3\%.

Puesto que "la vocación no nace sino que se hace", es cierto que la mujer científica ha recorrido un camino importante, ya no son consideradas bichos raros, pero también es verdad que en la actualidad el número de mujeres que alcanza una posición directiva es muy bajo (Salas, 1999). La investigación científica aún muestra discriminación sexual. Las mujeres ocupan los escalones más bajos de la pirámide laboral, además están ocupando puestos considerados socialmente feminizados, han pasado de estar excluidas a estar segregadas en determinados ámbitos científicos ya que no participan en la misma medida en todas las disciplinas (discriminación horizontal o territorial) y, cuando lo hacen, se quedan en los niveles inferiores (discriminación vertical o jerárquica), incluso van perdiendo terreno en algunos campos a medida que éstos incrementan su prestigio. (Pérez Sedeño, 2000a). Paralelamente, debido a que aún las mujeres se encuentran con la problemática de conciliación entre la vida profesional y

${ }^{23}$ En el curso $1977-78$ el $17.9 \%$ de la docencia universitaria era femenina. 
El lado oscuro de la mujer en la investigación científica...

la vida personal, su movilidad y dedicación se tornan impedidas; pues persisten mecanismos implícitos de segregación (González, 2002), para los que se han de buscar estrategias que erradiquen dichos mecanismos y terminen con ambos tipos de discriminación.

Si bien las mujeres han logrado grandes conquistas cuestionando el conocimiento que les venía dado desde una perspectiva androcéntrica, aún chocan con “techos invisibles" debido a su escasa presencia en altos niveles de decisión (Ballarín, 2001). La ciencia sigue siendo una empresa masculina, pero si las mujeres continuamos la andadura, la comunidad científica pronto nos dará la oportunidad de formar parte del espacio para el que muchas están capacitadas. Tenemos ante nosotras un panorama alentador, en el que una vez que se den las transformaciones necesarias, no tendremos que renunciar a ser mujer para dedicarnos a la tarea científica. 


\section{BIBLIOGRAFÍA}

Aвbott, P. y Wallace, C. (1990): An Introduction to Sociology: Feminist Perspectives. Londres: Routledge.

Agudo, Y. (2003): "Redes Universitarias Europeas: el futuro de los estudios de género", en las Jornadas Redes Internacionales de Mujeres, Redes Europeas de Excelencia, celebradas en la Facultad de Ciencias Políticas y Sociología, Madrid: UNED

-(2004): "La sombra femenina de la Ciencia" en El lado oscuro de la Modernización, XV Cursos de Verano UNED-2004. Ávila: Fundación Cultural Santa Teresa.

Aguinaga, J. (2004): El precio de un hijo. Los dilemas de la maternidad en una sociedad desigual. Barcelona: Debate.

AlBERDI, I. (1999): “El significado del género en las ciencias sociales”. En Política y Sociedad; Género y Ciencias Sociales, n 32, pp. 9-21.

Alic, M. (1991): El legado de Hipatia: Historia de las mujeres en la Ciencia desde la Antigüedad hasta fines del siglo XIX. Madrid: Siglo XXI editores.

BALlARÍN, P. (2001): La educación de las mujeres en la España contemporánea (siglos $X I X-X X)$. Madrid: Editorial Síntesis.

BARNES, B. (1987): Sobre Ciencia. Barcelona. Editorial Labor.

BORJA, M. (1970): Carreras y sexos. Barcelona: editorial Nova Terra.

CAMPS, V. (1998): El siglo de las mujeres. Madrid: Ediciones Cátedra.

CSIC (2001): Estudio sobre mujeres investigadoras del CSIC, disponible en http://www.csic.es $(20 / 06 / 03)$

- (2003): Mujeres investigadoras del CSIC. Informe elaborado por la Subdirección General de Recursos Humanos del CSIC, disponible en http://www.csic.es

- (2005): Informe mujeres investigadoras 2005. Informe elaborado por la Subdirección General de Recursos Humanos del CSIC, disponible en http://www.csic.es 
El lado oscuro de la mujer en la investigación científica...

DuRÁN, M. A. (1996): Mujeres y hombres en la formación de la teoría sociológica. Madrid: CIS.

- (1977): El trabajo de la mujer universitaria en la empresa. Madrid: Colección Forum Universidad Empresa.

ETAN (2001): Política científica de la Unión Europea. Promover la excelencia mediante la integración de la igualdad entre géneros. Informe del Grupo de Trabajo ETAN sobre las mujeres y la ciencia, Luxemburgo. Oficina de Publicaciones Oficiales de las Comunidades Europeas, disponible en http://www.cordis.lu/rtd2002/science-society/women.htm

FERnÁNDEZ, L. (2000): Roles de Género-Mujeres Académicas-¿Conflictos?, III Congreso Internacional Multidisciplinario sobre Mujer, Ciencia y Tecnología. Panamá: Universidad de Panamá.

FERNÁNDEZ, V. et al. (2002): “Ciencia y tecnología en el CSIC : una primera aproximación”. En Arbor: Ciencia, pensamiento y cultura, nº 679-680. Madrid: CSIC.

FLECHA, C. (1999): “Un espacio de libertad para las mujeres: su acceso a estudios universitarios”. En Mujer y Ciencia. Jaén: Universidad de Jaén.

FOX KÉLLER, E. (1991): Reflexiones sobre género y ciencia. Valencia: Alfons el Magnànim.

GARCÍA DE CORTÁZAR, M. y GARCÍA DE LEÓN, M.A. (1997): Mujeres en minoría. Una investigación sociológica sobre las catedráticas de universidad en España. Madrid: CIS, Opiniones y Actitudes, $n^{\circ} 16$, CIS.

- (codirectoras) (2001): Las académicas. (Profesorado universitario y género). Madrid: Instituto de la Mujer.

GonzÁlez, M. y PÉrez Sedeño, E. (2002): “Ciencia, Tecnología y Género”. En Para la educación de la Ciencia y la Cultura. Revista Iberoamericana de Ciencia, Tecnología, Sociedad e Innovación, OEI, $\mathrm{n}^{\mathrm{o}} 2$; disponible en: http://www.campus-oei.org/revistactsi/numero2/varios2.htm 
GuIL, A. (2002): "Investigadoras aquí y ahora: Universidad hispalense de Sevilla". En Entretejiendo Saberes. Congreso organizado por AUDEM en la Universidad de Sevilla.

HARDING, S. (1996): Ciencia y feminismo. Madrid: Ediciones Morata.

Instituto de la Mujer (1999): La otra mitad de la Ciencia. Madrid: Ministerio de Trabajo y Asuntos Sociales.

INE (2003): Boletín informativo del Instituto Nacional de Estadística: Cifras INE, disponible en www.ine.es

Kunn, T. S. (1962): The Structure of Scientific Revolutions. Chicago: The University of Chicago Press.

MArCo, B. (1999): "La emergencia de la mujer en la Ciencia. Algunos paradigmas de mujeres científicas". En Mujer y Ciencia. Jaén: Universidad de Jaén.

ORTIZ, T (1999): "Género y Ciencia" en Mujer y Ciencia. Jaén: Universidad de Jaén.

Pérez Sedeño, E. (2000a): “¿El poder de una ilusión?: Ciencia, Género y Feminismo)” en M.T. LÓPEZ DE LA VIEJA, (ed.): Feminismo: del pasado al presente. Salamanca: Ediciones Universidad de Salamanca.

- (2000 b): "Institucionalización de la ciencia valores epistemológicos y contextuales: un caso ejemplar". En Cadernos Pagu (15).

- (dir.)(2003): La Situación de las Mujeres en el Sistema Educativo de Ciencia y Tecnología en España y su Contexto Internacional. Programa de análisis y estudios de acciones destinadas a la mejora de la calidad de la enseñanza superior y de actividades del profesorado universitario. Instituto de FilosofíaCSIC, disponible en http://www.campus-oei.org/salactsi/EA2003-0031.pdf

SAlas, M. (1999): "La mujer y la ciencia: Mi propia historia". En Mujer y Ciencia. Jaén: Universidad de Jaén.

ScotT, J.W. (1990): "El género: una categoría útil para el análisis histórico" en Amelang, J. y Nash, M. (eds.). Historia y Género: Las mujeres en la Europa Moderna y Contemporánea. Valencia: Edicions Alfons el Magnànim. 
El lado oscuro de la mujer en la investigación científica...

SHIVA, V: (1995): Abrazar la vida. Mujer ecología y desarrollo. Madrid: Cuadernos inacabados (18), Horas y horas.

SOlsona I PAIRO, N. (1997): Mujeres cientificas de todos los tiempos. Madrid: TALASA Ediciones S.L.

VAN DEN EYNDE, A. (1994): “Género y ciencia, ¿términos contradictorios? Un análisis sobre la contribución de las mujeres al desarrollo científico". En Revista Iberoamericana de Educación. Género y Educación. Biblioteca Digital de la OEI: disponible en: http://www.campus-oei.org/oeivirt/rie06a03.htm

WoOlgar, S. (1992): Ciencia: abriendo la caja negra. Barcelona: Ánthropos.

\section{CONSULTAS ELECTRÓNICAS:}

http://www.campus-oei.org/revistactsi/numero2/varios2.htm

http://www.csic.es

http://www.ine.es

http://www.redtercermundo.org.uy

http://www.cordis.lu/rtd2002/science-society/women.htm

http://aupec.univalle.edu.co/informes/marzo98/mujerciencia.html 
